\title{
Data Science Guided Experiments Identify Conjugated Polymer Solution Concentration as a Key Parameter in Device Performance
}

\section{Supporting Information}

Rahul Venkatesh, ${ }^{\ddagger}$ Yulong Zheng, ${ }^{\dagger}$ Campbell Vierson, ${ }^{\dagger}$ Aaron $\mathrm{Liu}^{\ddagger}$, Carlos Silva, ${ }^{\dagger, \S, \|}$ Martha Grover, ${ }^{\ddagger, *}$ and Elsa Reichmanis Il, * $^{-}$

$\ddagger$ School of Chemical and Biomolecular Engineering, Georgia Institute of Technology, 311 Ferst Drive NW, Atlanta GA 30332, United States

†School of Chemistry and Biochemistry, Georgia Institute of Technology, 901 Atlantic Drive, Atlanta GA 30332, United States

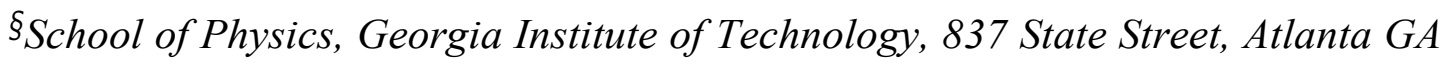
30332, United States

"School of Materials Science and Engineering, Georgia Institute of Technology, 771 Ferst Drive NW, Atlanta GA 30332, United States

"Department of Chemical \& Biomolecular Engineering, Lehigh University, 111 Research Drive W, Bethlehem PA 18015, United States

E-mail: elr420@lehigh.edu; martha.grover@chbe.gatech.edu 


\section{Dataset Details and Customized Classification Algorithm}

In this work, a dataset containing process-property information on OFET devices made from DPP-DTT was curated from 15 publications ${ }^{1-15}$. The database was curated on Excel, with each row containing a unique device obtained from published literatures, with the DOI, processing information and target performance metrics (hole mobility) for each device stored as columns. The dataset comprises of 115 OFET devices fabricated from neat DPP-DTT (92 devices) as well as a blend of DPP-DTT with an insulating matrix like PDMS, polystyrene and PCBM (23 devices). In this work, we only focus on the 92 OFET devices fabricated from neat DPP-DTT. 34 design variables/features were identified that were either numerical (21 features) or categorical (13 features). Examples of the numerical features include variables with continuous values such as polymer weight average molecular weight $\left(\mathrm{M}_{\mathrm{w}}\right)$, polydispersity (PDI), solution concentration, etc. The categorical features include solvent type, deposition type, annealing environment, etc. In this approach we focus on the numerical processing variables.

For mobility values presented as graphs in publications, a semi-automated tool called DataThief [www.datathief.org] was used to extract the mobility values up to three significant figures from the scatter plots. The average OFET hole mobility is the target performance metric that will be focused on in this work and the values reported in this dataset range from $8.5 \mathrm{~cm}^{2} / \mathrm{V}$.s to $1.0 \mathrm{x}$ $10^{-5}$, indicating the strong influence of the processing variables.

A custom classification algorithm developed by McBride et al. ${ }^{16}$ was applied to only the numerical processing variables to identify the most insightful features that would confine the "high" performing OFET devices to a reduced design region to gain new insights and guide future experiments. A schematic illustration of the classification approach is shown in Figure S1. 


\begin{tabular}{|c|c|c|c|c|c|c|}
\hline \multirow{8}{*}{ 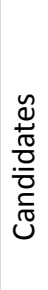 } & \multicolumn{4}{|c|}{ Design Variables } & \multicolumn{2}{|c|}{ Response } \\
\hline & DV 1 & DV 2 & $\ldots$ & DV $n$ & Measurement & Class \\
\hline & 299 & 2 & & 5 & 8.5 & High \\
\hline & 290 & 5 & & 4.5 & 6 & High 4 \\
\hline & 91 & 4 & $\cdots$ & 4 & 2 & High \\
\hline & 350 & 3 & & 2 & 0.8 & Low \\
\hline & 500 & 3 & & 3 & 0.05 & Low \\
\hline & 100 & 2 & & 10 & 0.0001 & Low \\
\hline
\end{tabular}

1. Sort observations and assign a classification threshold based on observed performance

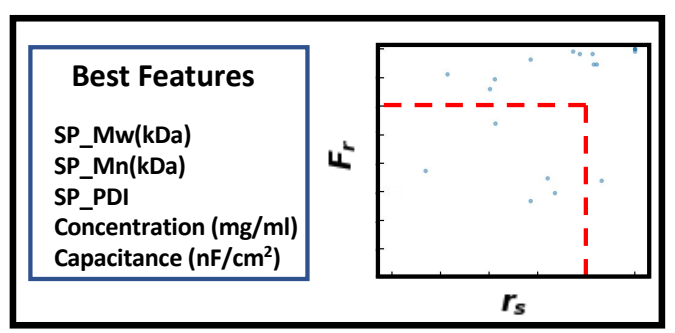

3. Select best features based on the calculated metrics

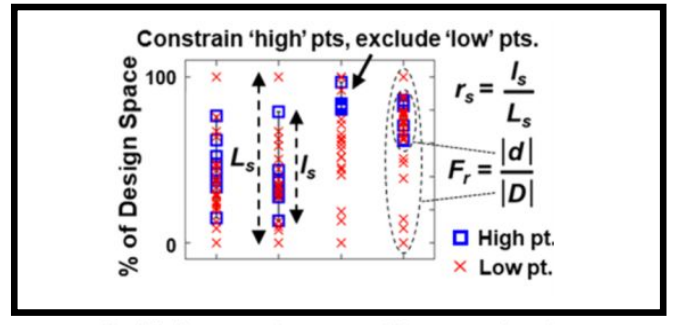

2. Using custom metrics, evaluate "quality" of descriptors based on how they constrain the high points to a reduced design region

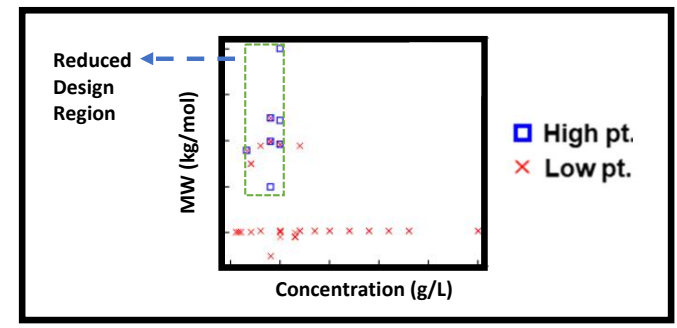

4. Analyze subset of best features and evaluate the reduced design region bounded by 'high' points

Figure S1. Schematic illustration of classification approach to select a smaller set of features that best separate "high" and "low" performers, and construct reduced design regions. Note the $1 D$ reduced design region in Step 2 is not representative of the actual dataset and was created for better visual representation. DV=Design Variables. Diagram adapted from Liu et al. (2020)

First, observations were sorted in decreasing order of average OFET hole mobility. A threshold of $1 \mathrm{~cm}^{2} / \mathrm{V}$.s was used to assign a label of "high" or "low" to each data point and this threshold can be adjusted (Figure S1, Box1). A total of 16 "high" devices and 76 "low" were identified. Then, a reduced design region was constructed for each continuous process design variable by calculating the upper and lower bounds of the region containing only the "high" points as shown in Figure S2. The quality of the reduced design region (RDR) for a specific feature was evaluated by considering two custom metrics that characterize the RDR relative to the total design space. The size reduction metric $r_{s}$ aids in searching for features in which the high points were constrained to a small fraction of the total design space. The metric $F_{r}$ accounts for nonuniformity of data density and finds features that exclude more low observations in the RDR. A visual interpretation of $r_{s}$ and $F_{r}$ for a design variable is illustrated in Figure S2. A feature with a low $r_{s}$ indicates that the "high" performing devices have been confined to small subset of the total design space. A feature with a low $F_{r}$ indicates that the reduced design space excludes more "low" performing devices. Processing variables with a low $r_{s}$ and low $F_{r}$ within a dataset are likely to be key indicators of better performance. 


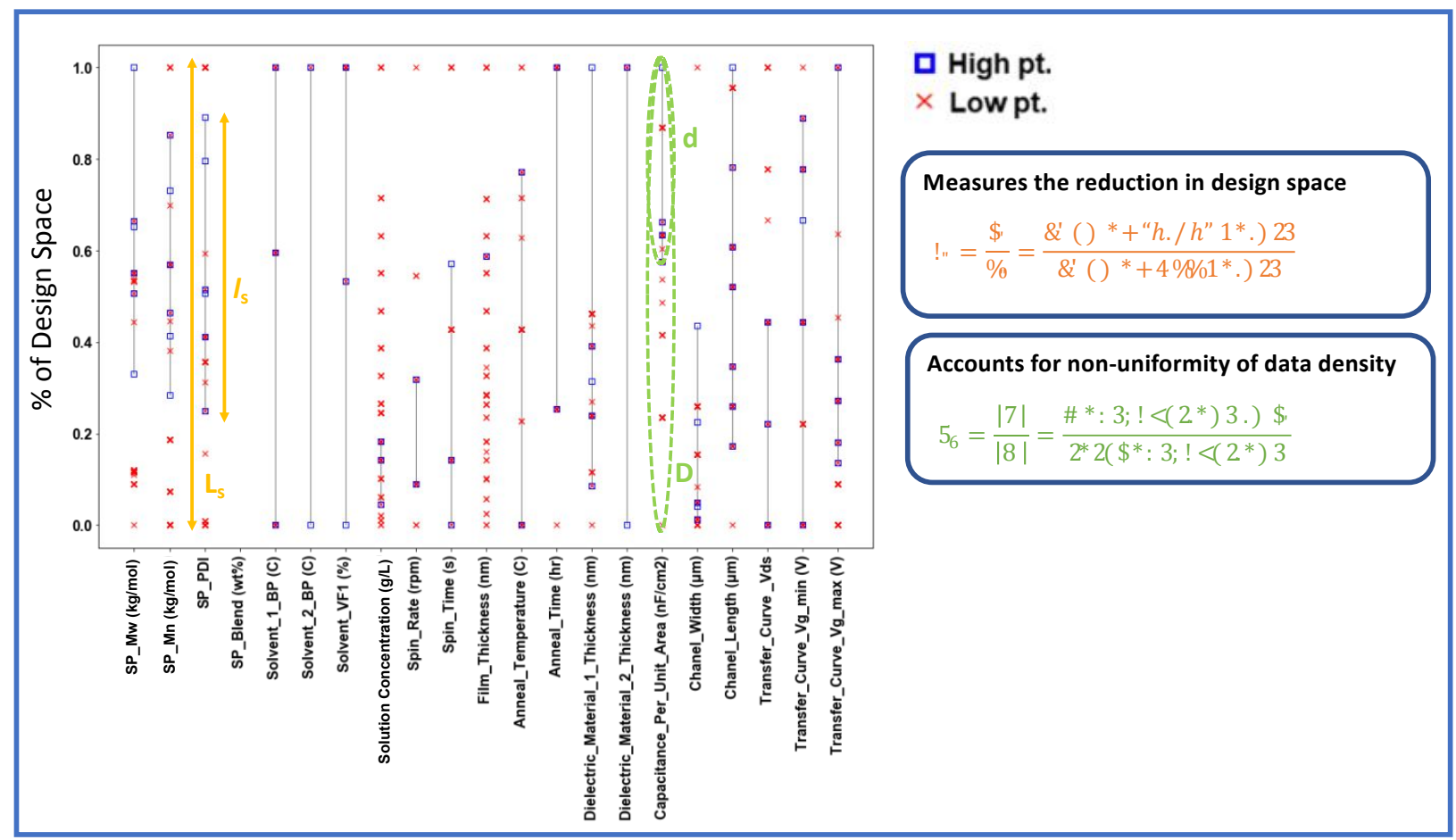

Figure S2. Classification approach of one-dimensional reduced design spaces containing OFET devices with hole mobility values exceeding $1 \mathrm{~cm}^{2} / V$.s. This figure corresponds to box 2 in Figure S1. Blue squares represent "high" performing data points which are equal to or above the cutoff of $1 \mathrm{~cm}^{2} / V . s$, red $x$ markers represent "low" points below the cutoff and the black lines represent the span of the reduced design region in the $1 D$ space.

To identify the most impactful features, the $r_{s}$ and $F_{r}$ values for each of the processing variables can be plotted against each other as shown in Figure S3. The best features were selected based on an arbitrary cut-off criteria $\left[r_{s} \& F_{r}<0.8\right]$ which was governed by domain expertise and visual inspection of the separation within the classification metrics. The feature selection approach was integrated with bootstrapping and cross-validation to find a subset of best-performing features as shown in the Figure S3. These selected features were then analyzed carefully to gain new insights. 


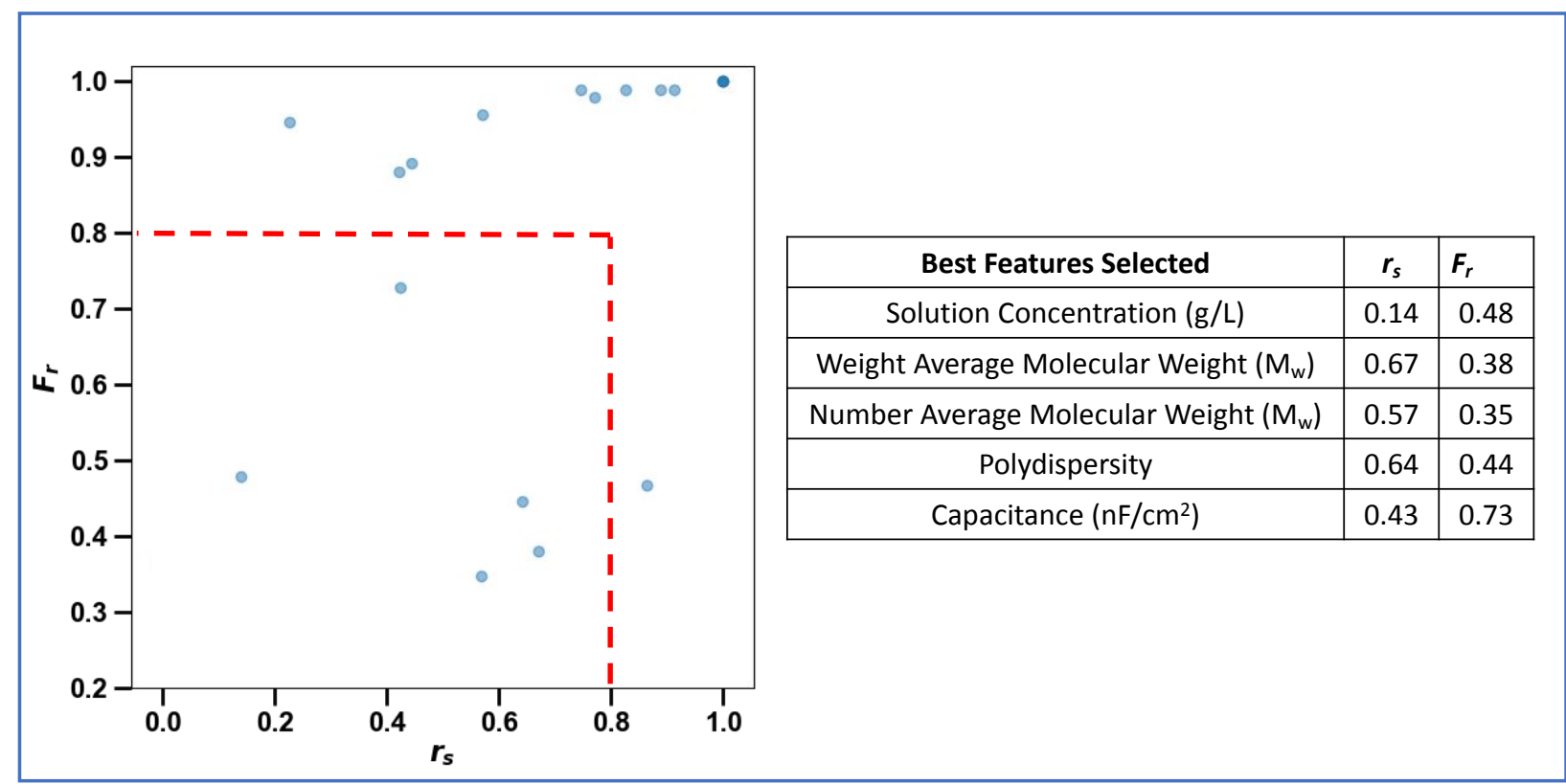

Figure S3. Custom metrics $r_{S}$ and $F_{r}$ for each numerical processing variable plotted against each other to identify the features/variables that confine the "high" performing points to a reduced design region. This figure corresponds to box 3 in Figure S1. An arbitrary threshold of $r_{S}$ and $F_{r}<0.8$ was selected for both metrics and this was governed by domain expertise and visual inspection of the separation within the classification metrics.

\section{Investigating "High" performing devices}

Total of 16 "high" performing devices from 5 different publications

- 10 of the 16 'high' performing devices are from the same publication

- 2 out of the 5 publications are from the same research group

Similarities in processing conditions between the high performing devices :

- Majority of the 'high' performing devices used chlorobenzene as the solvent. 13 of the "high" performing devices were processed using chlorobenzene as solvent, 2 using chloroform and 1 using Dichlorobenzene

- Majority of the 'high' performing devices used a concentration of $5 \mathrm{~g} / \mathrm{L} .10$ of the "high" performing devices were made from a concentration of $5 \mathrm{~g} / \mathrm{L}, 5$ devices were made from a concentration of $4 \mathrm{~g} / \mathrm{L}$ and 1 was made from a concentration of $2 \mathrm{~g} / \mathrm{L}$

- The solution processing environment for all 16 ' $\mathrm{high}$ ' performing devices were performing under ambient conditions

- Majority of the 'high' performing devices used N-doped SI as the substrate. 13 devices had N-doped SI as substrate, 1 had PET and 1 was unknown.

- Majority of the 'high' performing devices had source drain channels made out of gold. 14 devices had source/drain channels as $\mathrm{Au}, 2$ were unknown.

- Majority of the 'high' performing devices used spin coating as the method for film deposition. 14 devices used spin coating for deposition, 1 inkjet and 1 blade coating.

- Majority of the 'high' performing devices had films deposited at ambient conditions. 15 devices had films deposited in ambient conditions and 1 was unknown.

- All of the 'high' performing devices used the saturation regime to extract the mobility.

The Excel database along with the python code for the classification algorithm and figure 
plotting is available for download at :

https://github.com/rvenkatesh97/Reduced Design Region Classification DPPDTT OFETs

\section{OFET Fabrication and Characterization}

OFETs were used for the electrical characterization of the semiconducting DPP-DTT thin films made from different solution concentrations. Heavily n-doped silicon substrates with $300 \mathrm{~nm}$-thick thermally grown $\mathrm{SiO}_{2}$ dielectric layer were purchased from Rogue Valley Microdevices. The transistors functioned in a bottom gate bottom contact configuration with the n-doped silicon serving as a gate electrode and the $\mathrm{SiO}_{2}$ serving as a dielectric/insulating layer. The source and drain electrodes were fabricated via a standard photolithography liftoff process followed by E-beam evaporation (Denton Explorer) of $\mathrm{Cr}(3 \mathrm{~nm})$ as an adhesion layer and $\mathrm{Au}(50 \mathrm{~nm})$ onto the $\mathrm{SiO}_{2}$ surface. After electrode deposition, the substrates were then immersed in Acetone and the container was placed in an ultrasonic bath $(15 \mathrm{~min})$. This ultrasonication process was then repeated with methanol and IPA followed by sequential rinsing with acetone, methanol and IPA to wash off excess gold deposited on the metal. After drying the substrates with a nitrogen gun, they were then cleaned in a UV-ozone cleaner (Novascan PSD-UV) for 30 minutes to get rid of any excess photoresist and organic impurities. The cleaned substrates were then immersed in a 0.1 M OTS-18 solution in toluene overnight inside the glove box. The substrates were then sonicated in toluene the following day for 15 minutes followed by sequential rinsing with toluene, acetone, methanol and IPA and were blow dried with nitrogen gas. The semiconducting layer was then deposited onto the substrates using the solutions prepared as above via wire-bar coating at a speed of $10 \mathrm{~cm} / \mathrm{s}$ on a heated stage $\left(56^{\circ} \mathrm{C}\right)$. The substrates were then annealed at $56^{\circ} \mathrm{C}$ for 10 min to get rid of excess solvent.

OFET properties were measured in a nitrogen glovebox using an Agilent 4155C semiconductor parameter analyzer. The charge carrier mobility and threshold voltage were calculated in the saturation regime $\left(\mathrm{V}_{D S}=-80 \mathrm{~V}\right)$ by fitting the equation to the transfer plots of the drain current $\left(\mathrm{I}_{D S}\right)$ versus the gate voltage $\left(\mathrm{V}_{G S}\right)$.

$I_{D S}=\frac{W C_{i}}{2 L} \mu\left(V_{G}-V_{T}\right)^{2} \quad \mu_{s a t}=\frac{2 L}{C_{i} W}\left(\frac{\mathrm{d} \sqrt{I_{D L}}}{\mathrm{~d} V_{G}}\right)^{2}$

In the above equations, $\mathrm{W}$ is the channel width $(2 \mathrm{~mm}), \mathrm{L}$ is the channel length $(50 \mu \mathrm{m})$, $\mathrm{C}_{O X}$ is the capacitance per unit area of the $\mathrm{SiO}_{2}$ dielectric layer $\left(1.15 \times 10^{-8} \mathrm{~F} \mathrm{~cm}^{-2}\right), \mu$ is the hole mobility and $\mathrm{V}_{T}$ is the threshold voltage. The results in this work were averaged over 8-12 OFET devices for each concentration. 


\section{Franck Condon Fits}
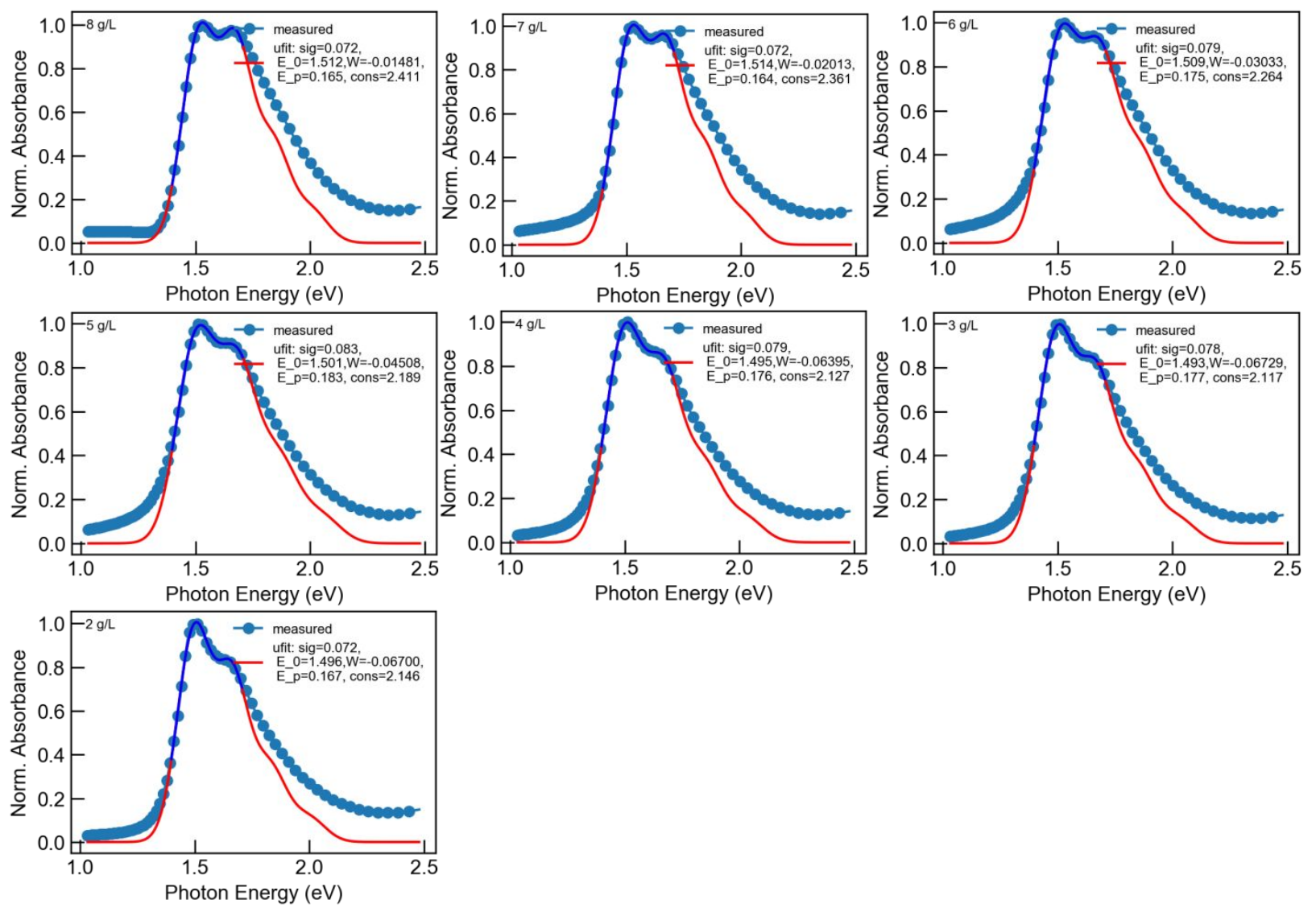

Figure S4. Modified FC fit for all absorption spectra at different concentrations along with the fit parameter 


\section{Microscopy Images and Profilometry Results}

(a)

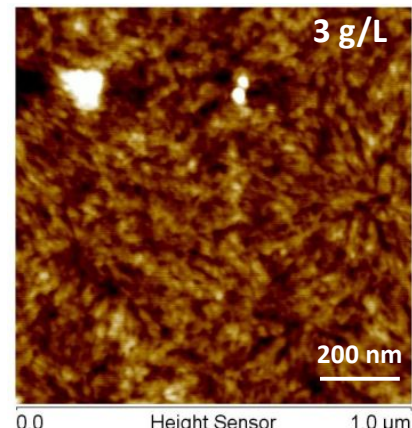

(b)

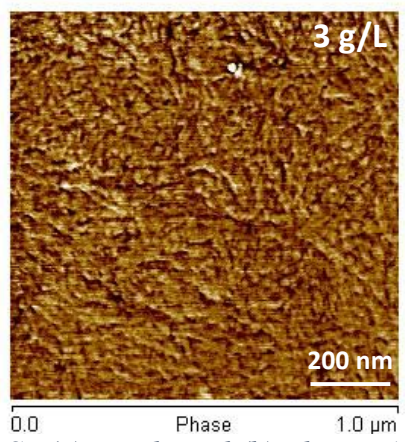

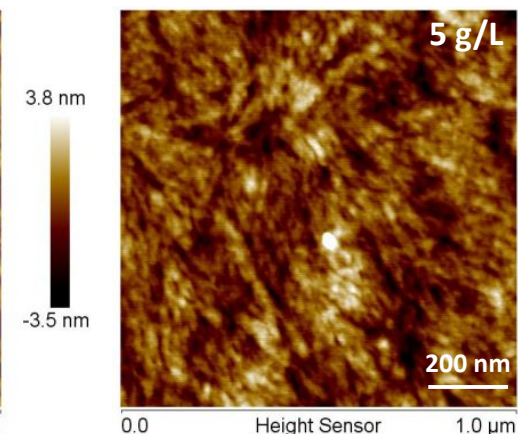
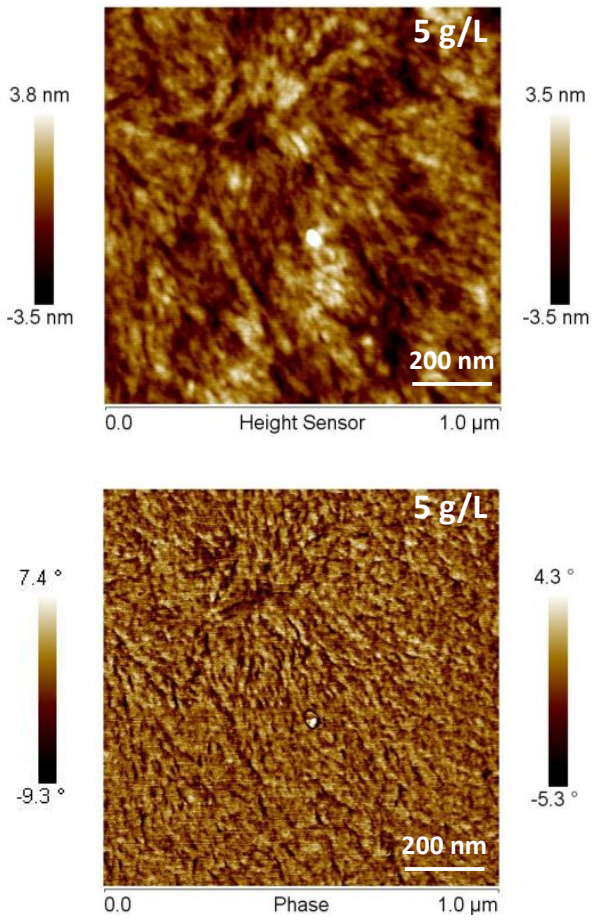
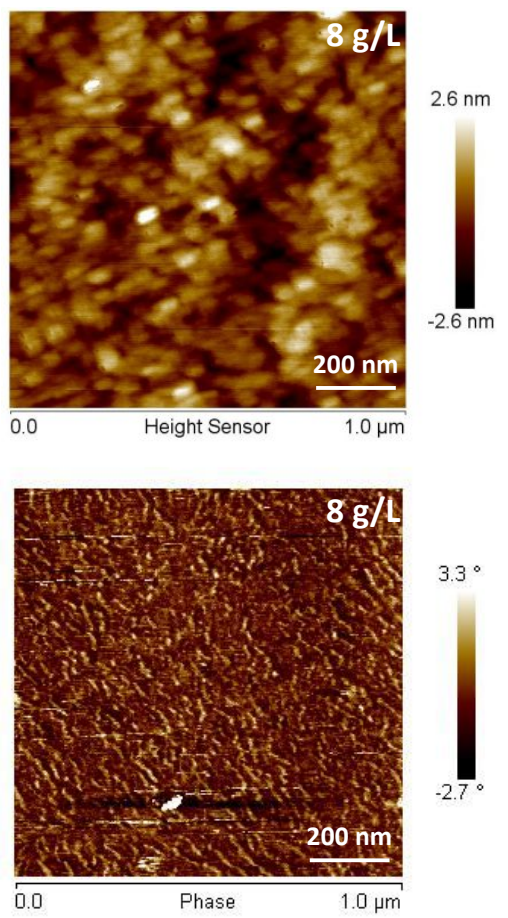

Figure S5.(a) Height and (b) Phase, Atomic Force Microscopy images of the DPP-DTT thin film samples prepared from solutions of different concentrations.

The AFM height and phase images (Figure S5) indicate fibrillar structures are formed in films prepared from more dilute concentrations which then disappear as solution concentration increases and the films ultimately becomes featureless.

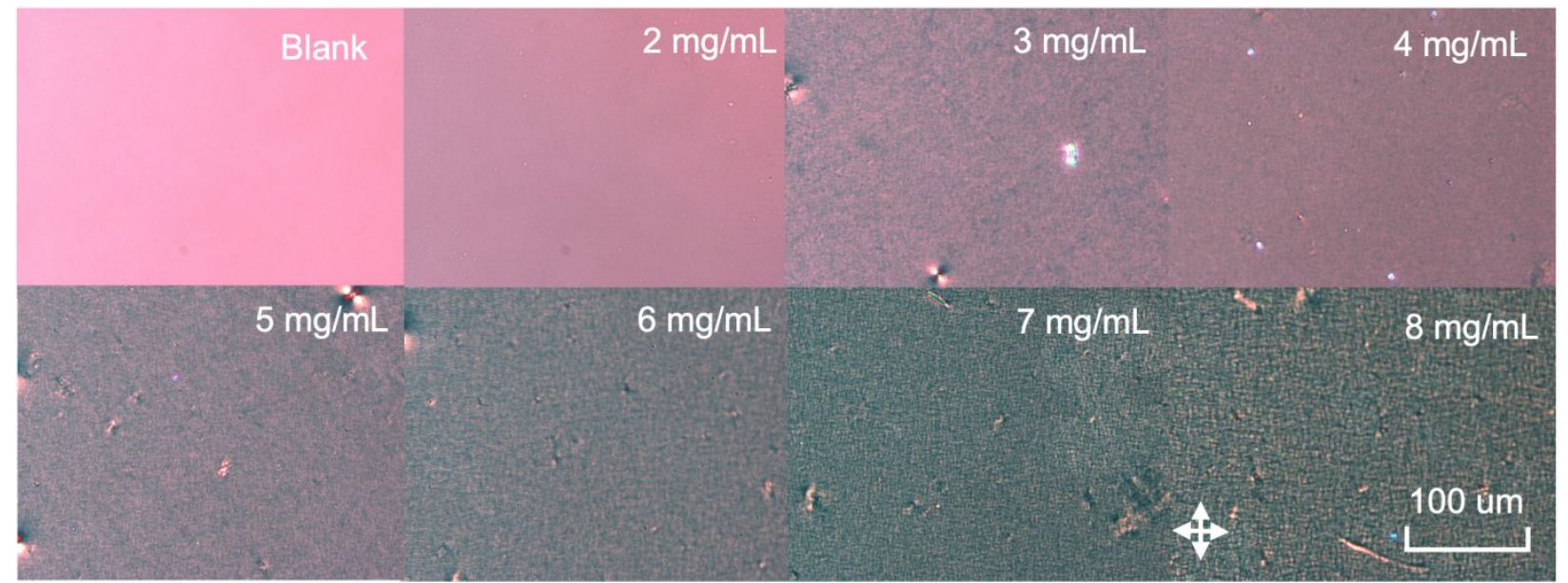

Figure S6. The cross-polarized optical microscopy images of the samples prepared from solutions of different concentrations. The directions of the polarizations are indicated with the cross signs. 
The CPOM images (Figure S6) indicate a clear change in intensity from low to high concentration, likely due to changes in film thicknesses. It is worth mentioning, that the noisy dots in the background of the CPOM images might be attributed to disordered aggregates, which increase in thickness with increasing concentration.

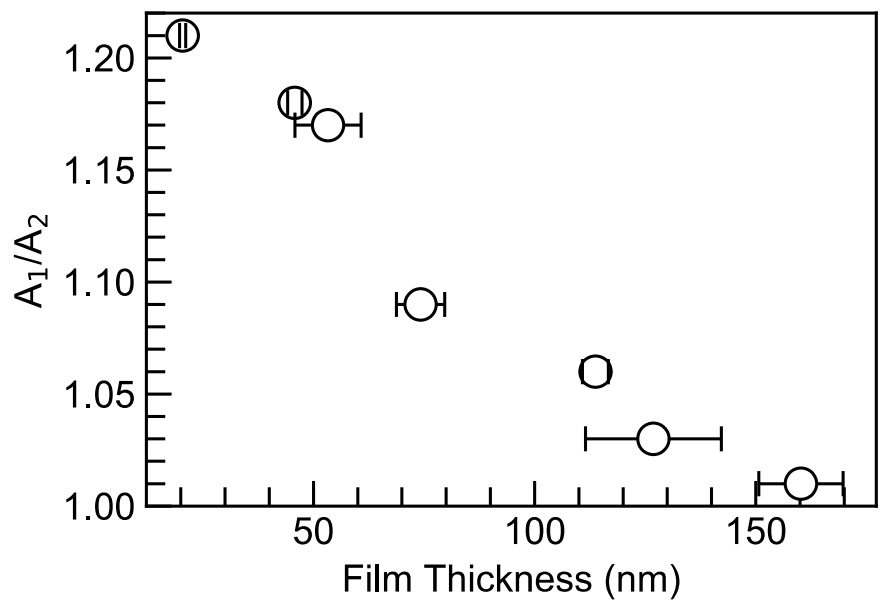

Figure S7.Film thickness of samples of 2 to $8 \mathrm{~g} / \mathrm{L}$ in chlorobenzene measured from profilometry as a function of $A_{1} / A_{2}$. The error bars represent $95 \%$ confidence interval averaged over 3 different points on the film. 


\section{OFET output and transfer characteristics}
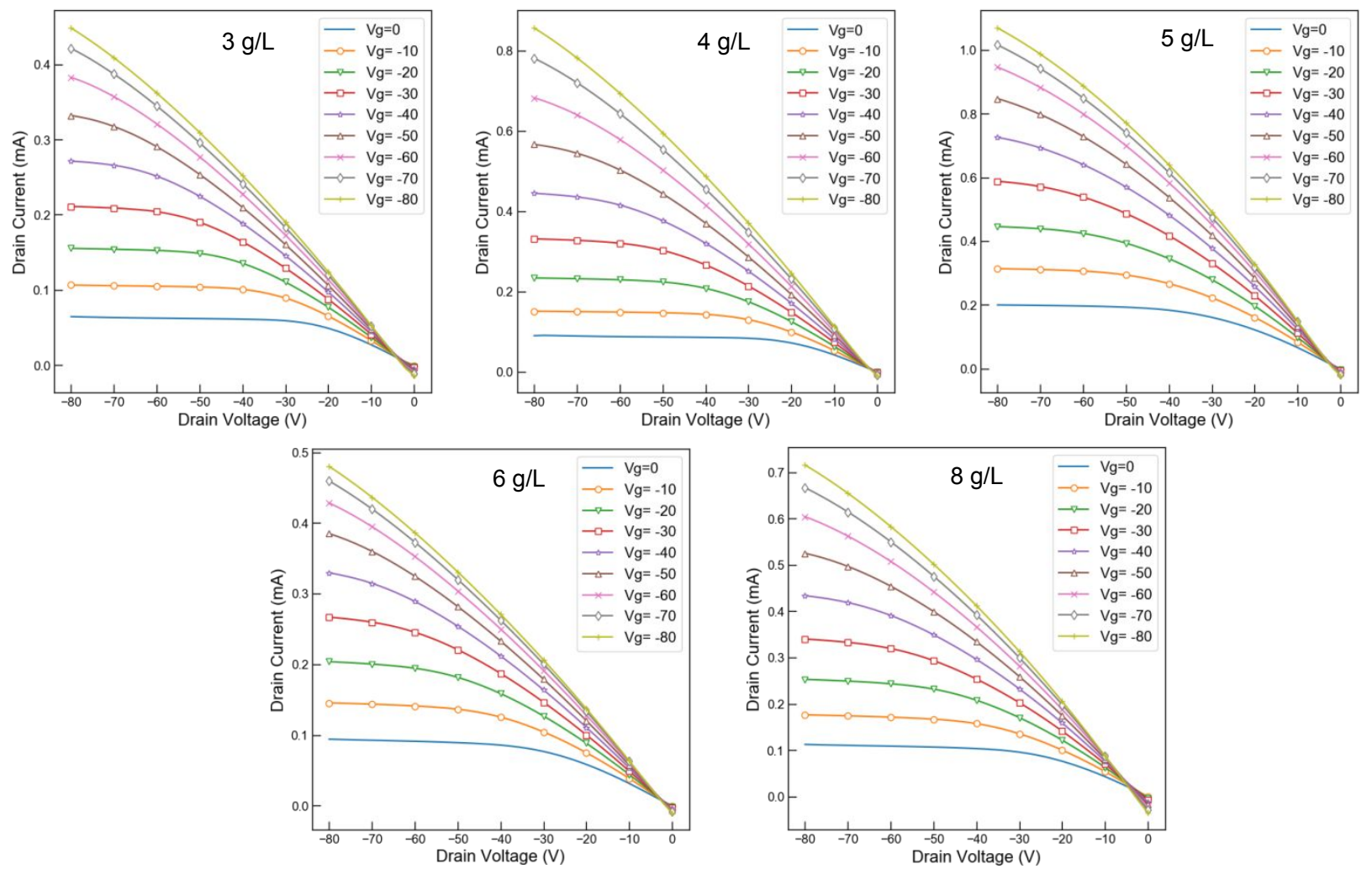

Figure S8. Output Characteristics for DPP-DTT thin films as a function of solution concentration averaged over 8-12 FET devices 

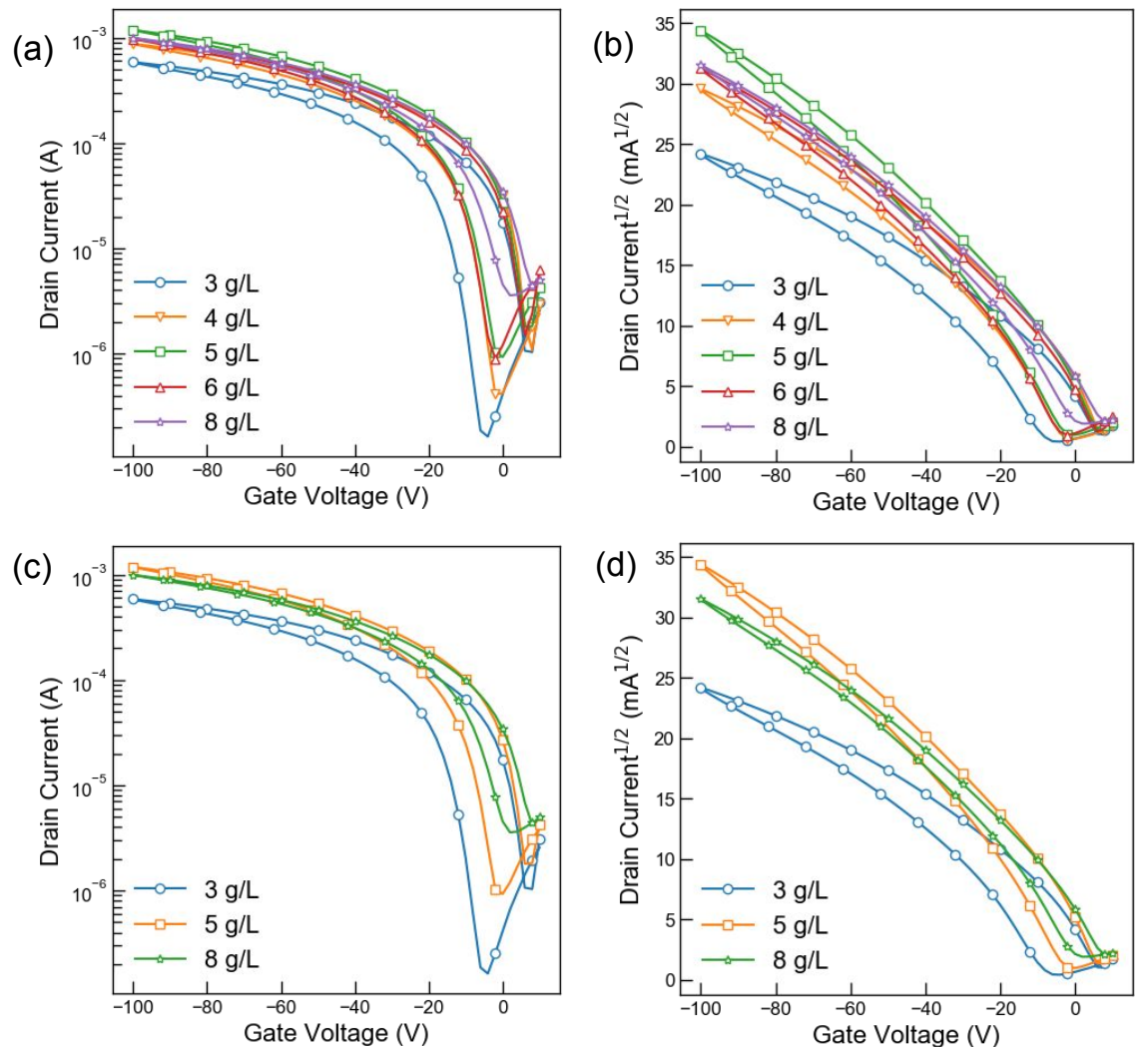

Figure S9. (a) and (b) Transfer characteristics at concentrations of 3, 4, 5, 6 and $8 \mathrm{~g} / \mathrm{L}$ exhibiting hysteresis. (c) and (d) Same transfer characteristics as in (a) and (b) but only for three concentrations for clearer visualization.

\section{Explanations for Deviations from Ideal Behavior}

Device characteristics exhibit hysteresis with the current being higher on the forward sweep towards larger ON voltages than on the reverse sweep as shown in figure S9. This behavior is likely due to the current in the OFET degrading during the timescale of the electrical measurement as a result of dispersive transport or charge trapping in the organic semiconductor or at the interface ${ }^{18}$. 


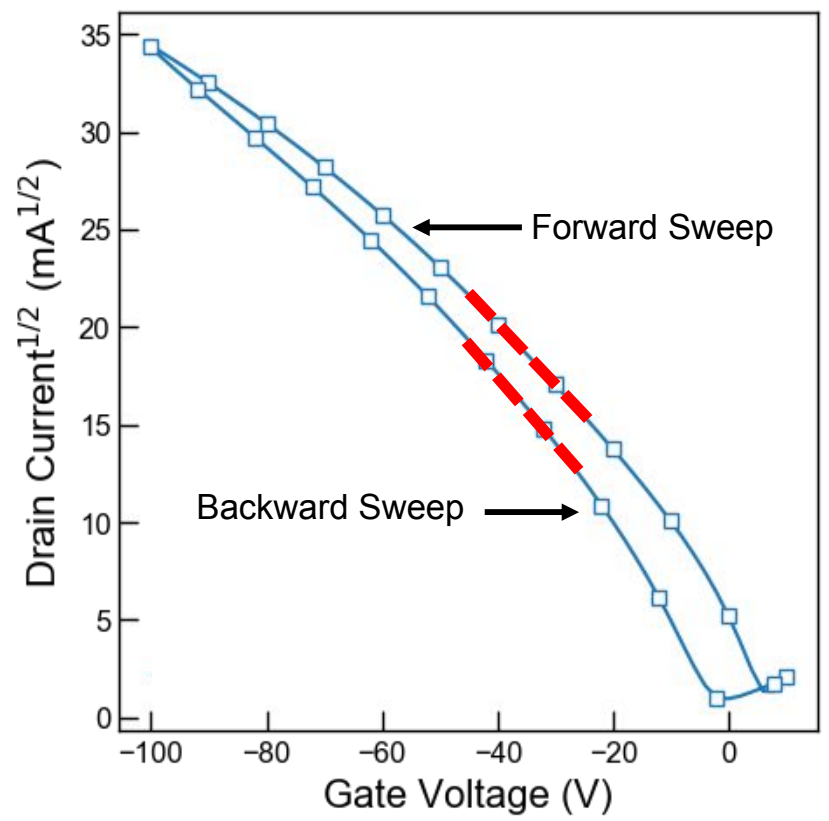

Figure S10. Backward and forward transfer characteristics $\left(I_{D}{ }^{1 / 2}-V_{G}\right)$ exhibiting regions of different slopes at lower and higher gate voltages. The mobility and threshold voltage for the forward and backward sweeps were extracted by fitting the saturation equation to the red dashed line ranging from $V_{G}=-45$ to $-25 \mathrm{~V}$. This range was selected based on the output characteristics in order to ensure we are in the saturation regime.

For the $\mathrm{I}^{1 / 2} \mathrm{~d}^{-} \mathrm{V}_{\mathrm{g}}$ curves, two regions of different slopes at lower and higher gate voltages were observed. This behavior has been observed previously for other high mobility D-A copolymers in bottom gate OFETs with self-assembled monolayer modified $\mathrm{SiO}_{2}$ gate dielectric ${ }^{19-23}$. Sirringhaus et al suggested that this behavior can be attributed to contact resistance effects, where the current becomes more contact-limited at higher gate voltages and the interfacial accumulation layer becomes more conducting ${ }^{24}$. Another possible explanation is that at higher gate voltages, charges from the accumulation layer are injected into the dielectric or interface andbecome trapped ${ }^{18,25}$. As recommended by McCulloch et $a l^{25}$ and Sirringhaus et al ${ }^{18}$ the low slope linear region, depicted by the red line in Figure S9 in SI, was used to extract a more conservative charge carrier mobility that is closer to the true value. 


\section{Forward Sweep Transfer Characteristics}

(a)

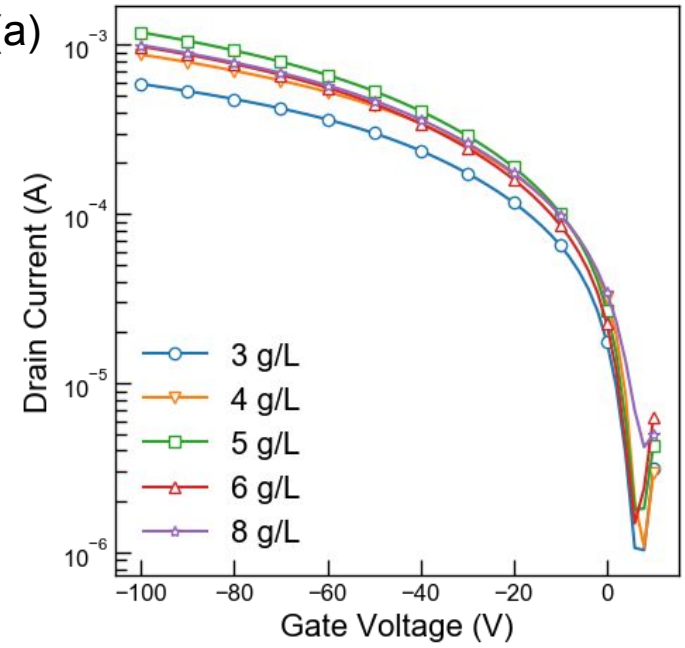

(b)

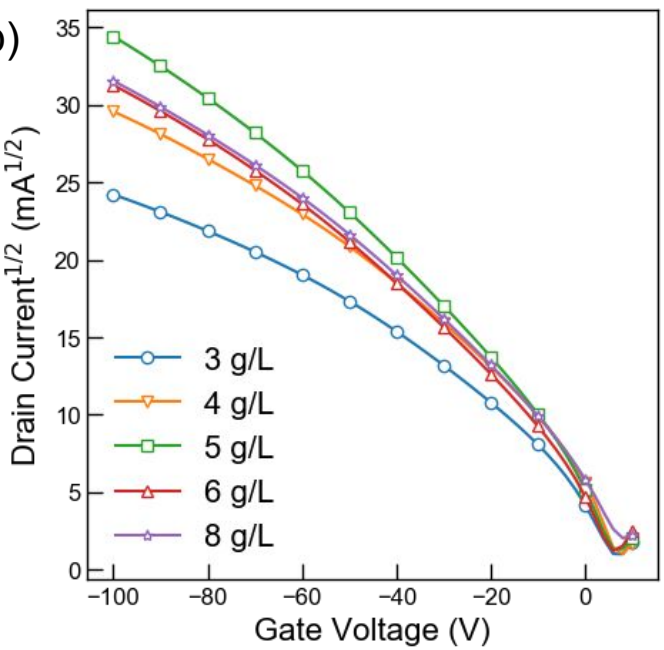

Figure S11. Forward sweep transfer characteristics $\left(V_{D S}=-80 \mathrm{~V}\right)$ as a function of solution concentration.
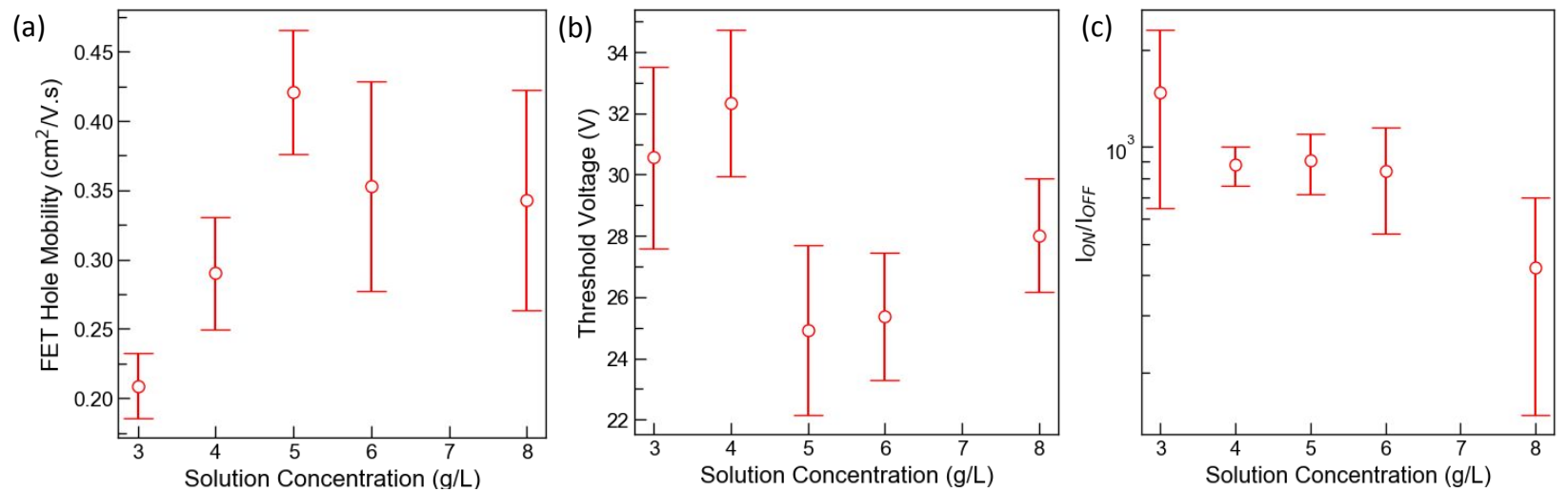

Figure S12. (a) and (b) FET hole mobility and threshold voltage extracted from the low slope region of the forward sweep transfer curve $\left(I_{D}{ }^{1 / 2}-V_{G}\right)$ as a function of solution concentration. (c) $I_{o n} / I_{\text {off }}$ obtained from the forward sweep transfer curve ( $I_{D}-$ $\left.V_{G}\right)$ as a function of solution concentration. The errors bars represent 95\% Confidence interval averaged over 8-12 OFET devices. 

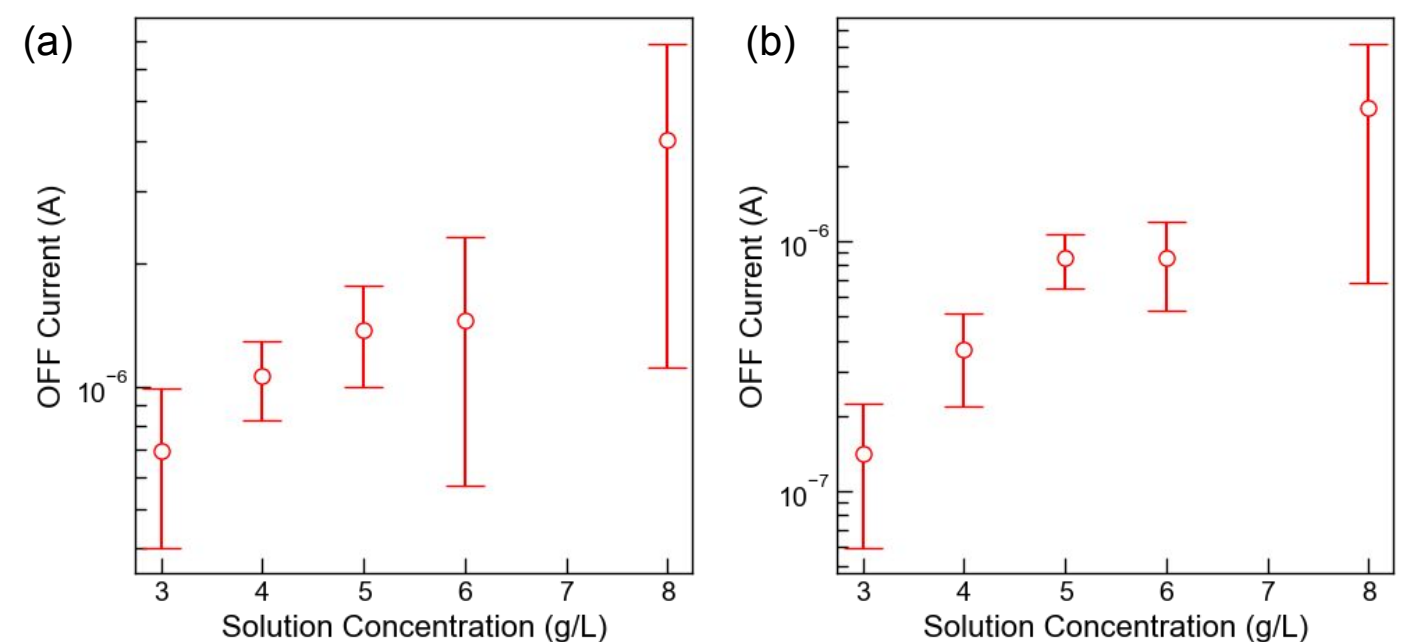

Figure S13. OFF current values obtained from (a) forward sweep and (b) backward sweep transfer characteristics $\left(I_{D}-V_{G}\right)$ as a function of solution concentration. The errors bars represent 95\% Confidence interval averaged over 8-12 OFET devices.

\section{Temperature Dependent DPP-DTT Solution State UV-Vis Spectra}

(a)

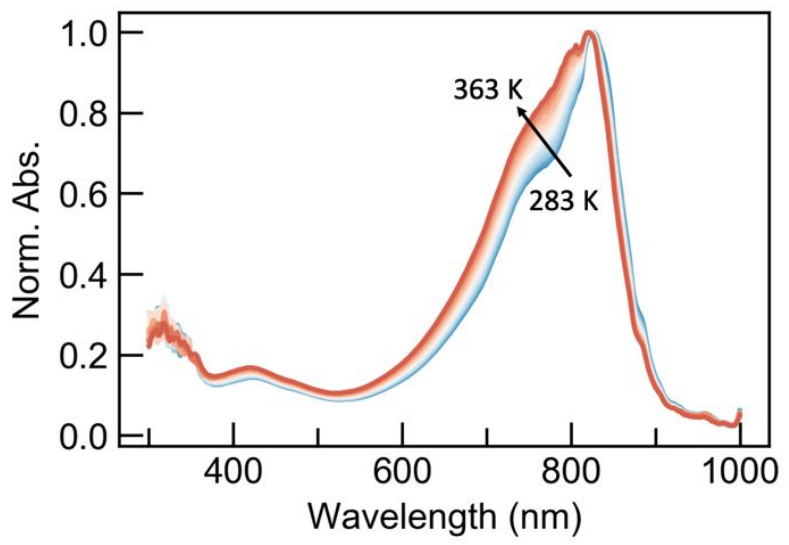

(b) $\quad 2 \mathrm{~g} / \mathrm{L}$ solution

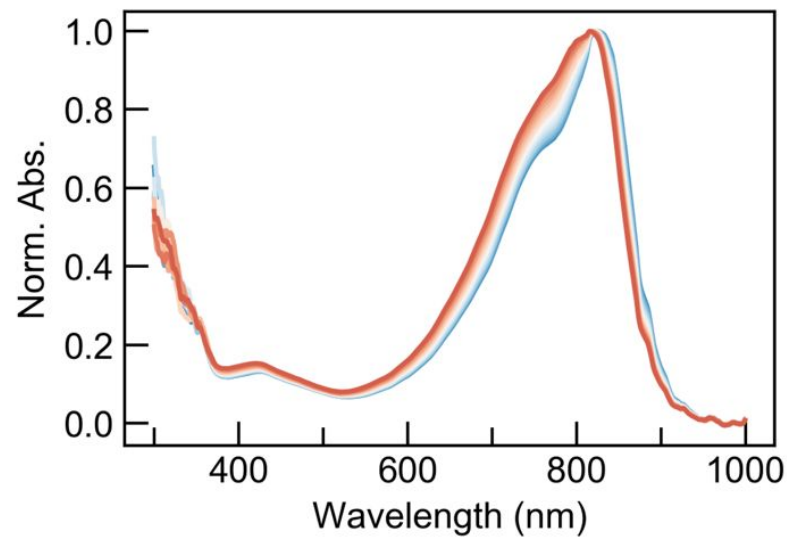

Figure S14. Solution-state absorption spectra of 2 and $8 \mathrm{~g} / \mathrm{L}$ DPP-DTT-CB solution measured from $283 \mathrm{~K}$ to $363 \mathrm{~K}$ with an $5 \mathrm{~K}$ temperature interval. 


\section{Analysis of P3HT dataset - Solution Concentration}

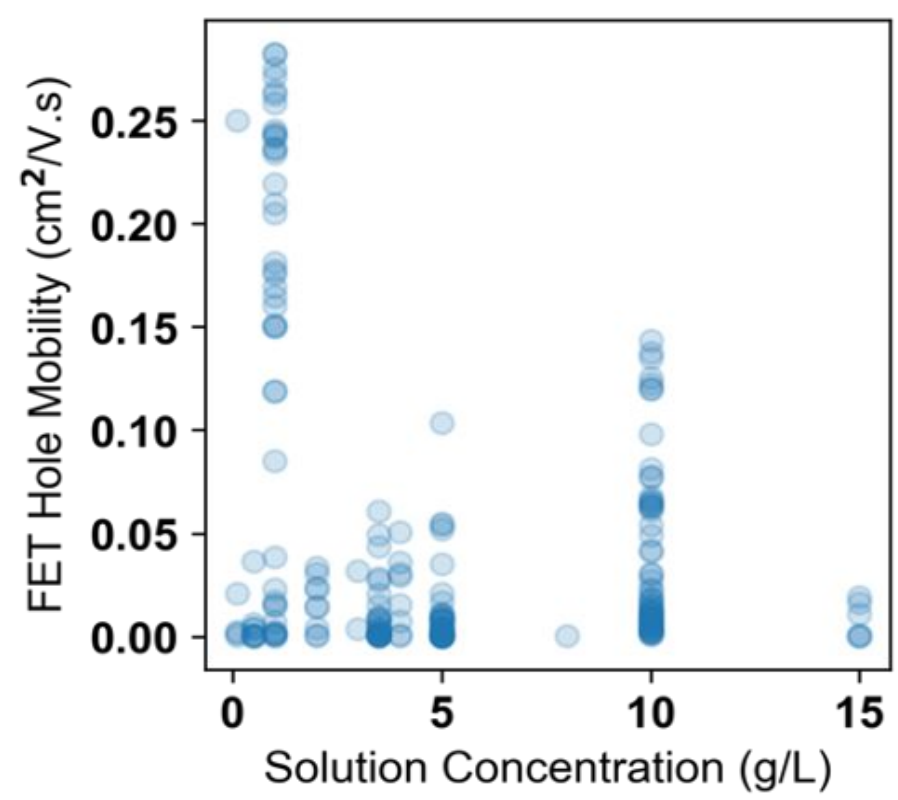

Figure S15. FET hole mobility versus solution concentration $(\mathrm{g} / \mathrm{L})$ for 202 P3HT devices across the literature. "High" performing devices have a mobility greater than $0.1 \mathrm{~cm}^{2} / V . s$. The optimum solution concentration range selected by the classification algorithm is from $1-10 \mathrm{~g} / \mathrm{L}$. 


\section{References}

1. Afzal, T.; Iqbal, M. J.; Iqbal, M. Z.; Sajjad, A.; Raza, M. A.; Riaz, S.; Kamran, M. A.; Numan, A.; Naseem, S., Effect of post-deposition annealing temperature on the charge carrier mobility and morphology of DPPDTT based organic field effect transistors. Chemical Physics Letters 2020, 750, 137507.

2. Armin, A.; Wolfer, P.; Shaw, P. E.; Hambsch, M.; Maasoumi, F.; Ullah, M.; Gann, E.; McNeill, C. R.; Li, J.; Shi, Z.; Burn, P. L.; Meredith, P., Simultaneous enhancement of charge generation quantum yield and carrier transport in organic solar cells. Journal of Materials Chemistry C2015, 3 (41), 10799-10812.

3. Baek, S. W.; Ha, J.-W.; Yoon, M.; Hwang, D.-H.; Lee, J., Shellac Films as a Natural Dielectric Layer for Enhanced Electron Transport in Polymer Field-Effect Transistors. ACS Applied Materials \& Interfaces 2018, 10 (22), 18948-18955.

4. Iqbal, M. J.; Haq, H.; Riaz, S.; Raza, M. A.; Iqbal, M. Z.; Chaudhry, M. U.; Naseem, S., On the Operational, shelf life and degradation mechanism in polymer field effect transistors. Superlattices and Microstructures 2019, 126, 125-131.

5. $\quad$ Kafle, P.; Zhang, F.; Schorr, N. B.; Huang, K.-Y.; Rodríguez-López, J.; Diao, Y., Printing 2D Conjugated Polymer Monolayers and Their Distinct Electronic Properties. Advanced Functional Materials 2020, 30 (12), 1909787.

6. Lei, Y.; Deng, P.; Li, J.; Lin, M.; Zhu, F.; Ng, T.-W.; Lee, C.-S.; Ong, B. S., Solution-Processed Donor-Acceptor Polymer Nanowire Network Semiconductors For High-Performance Field-Effect Transistors. Scientific Reports 2016, 6 (1), 24476.

7. $\quad$ Lei, Y.; Wu, B.; Chan, W.-K. E.; Zhu, F.; Ong, B. S., Engineering gate dielectric surface properties for enhanced polymer field-effect transistor performance. Journal of Materials Chemistry C2015, 3(47), 12267-12272.

8. Li, J.; Zhao, Y.; Tan, H. S.; Guo, Y.; Di, C.-A.; Yu, G.; Liu, Y.; Lin, M.; Lim, S. H.; Zhou, Y.; $\mathrm{Su}, \mathrm{H}$.; Ong, B. S., A stable solution-processed polymer semiconductor with record high-mobility for printed transistors. Scientific Reports 2012, 2 (1), 754.

9. Park, H.; Yoo, S.; Won, J. C.; Kim, Y. H., Room-temperature, printed, low-voltage, flexible organic field-effect transistors using soluble polyimide gate dielectrics. APL Materials 2020, 8(1), 011112.

10. Qu, G.; Zhao, X.; Newbloom, G. M.; Zhang, F.; Mohammadi, E.; Strzalka, J. W.; Pozzo, L. D.; Mei, J.; Diao, Y., Understanding Interfacial Alignment in Solution Coated Conjugated Polymer Thin Films. ACS Applied Materials \& Interfaces 2017, 9 (33), 27863-27874.

11. Sarkar, T.; Schneider, S. A.; Ankonina, G.; Hendsbee, A. D.; Li, Y.; Toney, M. F.; Frey, G. L., Tuning Intra and Intermolecular Interactions for Balanced Hole and Electron Transport in Semiconducting Polymers. Chemistry of Materials 2020, 32 (17), 7338-7346.

12. Xi, Y.; Wolf, C. M.; Pozzo, L. D., Self-assembly of donor - acceptor conjugated polymers induced by miscible 'poor' solvents. Soft Matter 2019, 15 (8), 1799-1812.

13. Xu, H.; Li, J.; Leung, B. H. K.; Poon, C. C. Y.; Ong, B. S.; Zhang, Y.; Zhao, N., A high-sensitivity near-infrared phototransistor based on an organic bulk heterojunction. Nanoscale 2013, 5 (23), 11850-11855.

14. Zhang, G.; Lee, S.; Gutiérrez-Meza, E.; Buckley, C.; McBride, M.; Valverde-Chávez, D. A.; Kwon, Y. H.; Savikhin, V.; Xiong, H.; Dunn, T. J.; Toney, M. F.; Yuan, Z.; Silva, C.; Reichmanis, E., Robust and Stretchable Polymer Semiconducting Networks: From Film Microstructure to Macroscopic Device Performance. Chemistry of Materials 2019, 31 (17), 6530-6539.

15. Zhang, G.; McBride, M.; Persson, N.; Lee, S.; Dunn, T. J.; Toney, M. F.; Yuan, Z.; Kwon, Y.-H.; Chu, P.-H.; Risteen, B.; Reichmanis, E., Versatile Interpenetrating Polymer Network Approach to Robust Stretchable Electronic Devices. Chemistry of Materials 2017, 29 (18), 7645-7652. 
16. McBride, M.; Persson, N.; Reichmanis, E.; Grover, M. A., Solving Materials' Small Data Problem with Dynamic Experimental Databases. Processes 2018, 6 (7), 79-96.

17. Liu, A. L.; Venkatesh, R.; McBride, M.; Reichmanis, E.; Meredith, J. C.; Grover, M. A., Small Data Machine Learning: Classification and Prediction of Poly(ethylene terephthalate) Stabilizers Using Molecular Descriptors. ACS Applied Polymer Materials 2020, 2 (12), 5592-5601.

18. Sirringhaus, H., 25th Anniversary Article: Organic Field-Effect Transistors: The Path Beyond Amorphous Silicon. Advanced Materials 2014, 26 (9), 1319-1335.

19. Chen, H.; Guo, Y.; Yu, G.; Zhao, Y.; Zhang, J.; Gao, D.; Liu, H.; Liu, Y., Highly $\pi$-extended copolymers with diketopyrrolopyrrole moieties for high-performance field-effect transistors. Adv Mater 2012, 24 (34), 4618-22.

20. $\quad$ Kang, I.; An, T. K.; Hong, J.-a.; Yun, H.-J.; Kim, R.; Chung, D. S.; Park, C. E.; Kim, Y.-H.; Kwon, S.-K., Effect of Selenophene in a DPP Copolymer Incorporating a Vinyl Group for High-Performance Organic Field-Effect Transistors. Advanced Materials 2013, 25 (4), 524-528.

21. Lei, T.; Dou, J.-H.; Pei, J., Influence of Alkyl Chain Branching Positions on the Hole Mobilities of Polymer Thin-Film Transistors. Advanced Materials 2012, 24 (48), 6457-6461.

22. Li, J.; Zhao, Y.; Tan, H. S.; Guo, Y.; Di, C.-A.; Yu, G.; Liu, Y.; Lin, M.; Lim, S. H.; Zhou, Y.; Su, H.; Ong, B. S., A Stable Solution-Processed Polymer Semiconductor with Record High-Mobility for Printed Transistors. Scientific Reports 2012, 2(1), 754-763.

23. Matthews, J. R.; Niu, W.; Tandia, A.; Wallace, A. L.; Hu, J.; Lee, W.-Y.; Giri, G.; Mannsfeld, S. C. B.; Xie, Y.; Cai, S.; Fong, H. H.; Bao, Z.; He, M., Scalable Synthesis of Fused ThiopheneDiketopyrrolopyrrole Semiconducting Polymers Processed from Nonchlorinated Solvents into High Performance Thin Film Transistors. Chemistry of Materials 2013, 25 (5), 782-789.

24. Braga, D.; Horowitz, G., High-Performance Organic Field-Effect Transistors. Advanced Materials 2009, 21, 1473-1486.

25. McCulloch, I.; Salleo, A.; Chabinyc, M., Avoid The Kinks when Measuring Mobility. Science 2016, 352 (6293), 1521. 